University of Wollongong

Research Online

Faculty of Engineering and Information

Faculty of Engineering and Information

Sciences - Papers: Part B

Sciences

2018

Total electron scattering cross sections from thiophene for the $(1-300 \mathrm{eV})$ impact energy range

A I. Lozano

Consejo Superior de Investigaciones Científicas

A Loupas

Universidade Nova de Lisboa

Francisco Blanco

Universidad Complutense de Madrid

J D. Gorfinkiel

Open University

Gustavo Garcia

University of Wollongong, garcia@uow.edu.au

Follow this and additional works at: https://ro.uow.edu.au/eispapers1

Part of the Engineering Commons, and the Science and Technology Studies Commons

Research Online is the open access institutional repository for the University of Wollongong. For further information contact the UOW Library: research-pubs@uow.edu.au 


\title{
Total electron scattering cross sections from thiophene for the (1-300 eV) impact energy range
}

\author{
Abstract \\ Experimental electron scattering cross sections for thiophene in the impact energy range from 1 to 300 \\ $\mathrm{eV}$ have been measured with a magnetically confined electron transmission-beam apparatus. Random \\ uncertainty limits have been estimated to be less than $5 \%$, and systematic errors derived from acceptance \\ angle limitations have also been identified and evaluated. Experimental values are compared with our \\ previous low energy (1-15 eV) R-matrix and intermediate/high energy (15-300 eV) IAM-SCAR+I \\ calculations finding reasonable agreement, within the combined uncertainty limits. Some of the low \\ energy shape and core-excited resonances predicted by previous calculations are experimentally \\ confirmed in this study.

\section{Disciplines} \\ Engineering | Science and Technology Studies

\section{Publication Details} \\ Lozano, A. I., Loupas, A., Blanco, F., Gorfinkiel, J. D. \& Garcia, G. (2018). Total electron scattering cross \\ sections from thiophene for the (1-300 eV) impact energy range. Journal of Chemical Physics, 149 (13), \\ 134303-1-134303-7.
}




\title{
Total electron scattering cross sections from thiophene for the (1-300 eV) impact energy range
}

\author{
A. I. Lozano, A. Loupas, F. Blanco, J. D. Gorfinkiel, and G. García
}

Citation: J. Chem. Phys. 149, 134303 (2018); doi: 10.1063/1.5050349

View online: https://doi.org/10.1063/1.5050349

View Table of Contents: http://aip.scitation.org/toc/jcp/149/13

Published by the American Institute of Physics

\section{Articles you may be interested in}

Cross sections for electron scattering from thiophene for a broad energy range

The Journal of Chemical Physics 149, 034304 (2018); 10.1063/1.5040352

Integral elastic, vibrational-excitation, electronic-state excitation, ionization, and total cross sections for electron scattering from para-benzoquinone

The Journal of Chemical Physics 148, 204305 (2018); 10.1063/1.5028298

Positron scattering from pyridine

The Journal of Chemical Physics 148, 144308 (2018); 10.1063/1.5024246

Multiphoton photoelectron circular dichroism of limonene with independent polarization state control of the bound-bound and bound-continuum transitions

The Journal of Chemical Physics 149, 134301 (2018); 10.1063/1.5042533

Fully anharmonic infrared cascade spectra of polycyclic aromatic hydrocarbons

The Journal of Chemical Physics 149, 134302 (2018); 10.1063/1.5038725

Spin-polarized and thermospin-polarized transport properties of phthalocyanine dimer based molecular junction with different transition metal atoms

The Journal of Chemical Physics 149, 134305 (2018); 10.1063/1.5047081

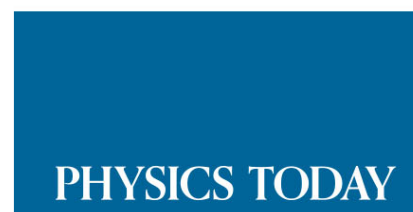

WHITEPAPERS
ADVANCED LIGHT CURE ADHESIVES

Take a closer look at what these environmentally friendly adhesive systems can do
READ NOW

PRESENTED BY

(8) MASTERBOND' 


\title{
Total electron scattering cross sections from thiophene for the (1-300 eV) impact energy range
}

\author{
A. I. Lozano, ${ }^{1,2, a)}$ A. Loupas, ${ }^{3,4}$ F. Blanco, ${ }^{5}$ J. D. Gorfinkiel, ${ }^{4}$ and G. García ${ }^{1,6, b)}$ \\ ${ }^{1}$ Instituto de Física Fundamental, Consejo Superior de Investigaciones Científicas, Serrano 113-Bis, \\ 28006 Madrid, Spain \\ ${ }^{2}$ Escuela de Doctorado de la UNED-Programa de Doctorado en Ciencias, 28015 Madrid, Spain \\ ${ }^{3}$ Laboratório de Colisões Atómicas e Moleculares, CEFITEC, Departamento de Física, Faculdade de Ciências \\ e Tecnologia, Universidade Nova de Lisboa, Campus de Caparica, 2829-516 Lisbon, Portugal \\ ${ }^{4}$ School of Physical Sciences, The Open University, Walton Hall, Milton Keynes MK7 6AA, United Kingdom \\ ${ }^{5}$ Departamento de Física Atómica, Molecular y Nuclear, Universidad Complutense de Madrid, 28040 Madrid, \\ Spain \\ ${ }^{6}$ Centre for Medical Radiation Physics, University of Wollongong, Wollongong, NSW, Australia
}

(Received 30 July 2018; accepted 17 September 2018; published online 2 October 2018)

\begin{abstract}
Experimental electron scattering cross sections for thiophene in the impact energy range from 1 to $300 \mathrm{eV}$ have been measured with a magnetically confined electron transmission-beam apparatus. Random uncertainty limits have been estimated to be less than $5 \%$, and systematic errors derived from acceptance angle limitations have also been identified and evaluated. Experimental values are compared with our previous low energy (1-15 eV) R-matrix and intermediate/high energy (15-300 eV) IAM-SCAR+I calculations finding reasonable agreement, within the combined uncertainty limits. Some of the low energy shape and core-excited resonances predicted by previous calculations are experimentally confirmed in this study. Published by AIP Publishing. https://doi.org/10.1063/1.5050349
\end{abstract}

\section{INTRODUCTION}

Electron interactions with complex molecules have been the subject of great interest in the last few years due to their relevance in important applications such as radiation damage ${ }^{1,2}$ and electron transport in plasmas $^{3}$ and condensed media. ${ }^{4}$ These applications require evaluated differential (DCS) and integral (ICS) cross section data over a broad energy range for which different theoretical and experimental techniques need to be applied and the consistency between the corresponding results needs to be verified. In the case of thiophene $\left(\mathrm{C}_{4} \mathrm{H}_{4} \mathrm{~S}\right)$, commonly used as an anti-inflammatory drug, we have recently calculated ${ }^{5}$ differential and integral elastic, integral inelastic and total electron cross sections over a broad energy range $(0.1-1000 \mathrm{eV})$ by combining the R-matrix procedure for the lower energies with the IAM-SCAR+I method for intermediate and high energies. Our previous theoretical results were compared with experimental and theoretical data available in the literature (see Ref. 5 and references therein). In particular, the lower energy data were found to be in agreement with previous Schwinger multichannel with pseudopotentials (SMCPP) calculations, ${ }^{6}$ and the consistency with the IAMSCAR+I higher energy results was found to be reasonably good. This provided a consistent picture of the scattering process in the whole energy range. However, an experimental validation of these cross section data is needed in order to ensure that they are appropriate for use in modelling and to establish some realistic uncertainty limits.

a)anita_ilm@iff.csic.es

b)g.garcia@csic.es
In this context, we present here absolute values for the total electron scattering cross section (TCS) from thiophene for impact energies ranging from 1 to $300 \mathrm{eV}$ measured with a state-of-the-art magnetically confined electron transmission-beam apparatus ${ }^{7}$ together with a detailed analysis of their associated random and systematic uncertainty sources. Since the TCS corresponds to the sum of the ICS related to all the open channels at a given energy, they are excellent reference values to carry out the aforementioned validation.

The remainder of this paper is structured as follows: In Sec. II, some details on the experimental setup and procedure are given. A brief description of the calculation procedures which are relevant to this study are summarised in Sec. III. The current experimental results are presented and discussed in Sec. IV and compared with the available theoretical data. Our conclusions are finally summarized in Sec. V.

\section{EXPERIMENTAL SETUP AND PROCEDURE}

The experimental apparatus and techniques used for the present transmission-beam attenuation study have recently been described ${ }^{7}$ and so will not be detailed again here. In brief, a linear electron beam is confined by an intense (typically $0.1 \mathrm{~T}$ ) axial magnetic field which converts any scattering event into a kinetic energy loss in the forward direction, i.e., parallel to the magnetic field (see Ref. 7 for full details). The primary electron beam, generated by an emitting filament, is cooled and confined in a magnetic nitrogen gas trap (GT) which reduces the initial energy spread of $500 \mathrm{meV}$ down to about $100-200 \mathrm{meV}$. Pulsed voltages applied to the trap 
electrodes produce a pulsed electron beam with well-defined energy and narrow energy spread to enter the scattering cell. The scattering chamber (SC) is a $40 \mathrm{~mm}$ long gas cell, defined by two $1.5 \mathrm{~mm}$ diameter apertures, through which the pulsed electron beam passes when the thiophene pressure inside the chamber is varied from 0 to 5 mTorr (as measured by a MKSBaratron 627B absolute capacitance manometer). Electrons emerging from the SC are analysed in energy by a retarding potential analyser (RPA) and finally detected by a double microchannel plate (MCP) electron multiplier operating in single counting mode. The total cross section $\left(\sigma_{\mathrm{T}}\right)$ is determined from the transmitted intensity, which follows the well-known Lambert-Beer attenuation law for ideal gases

$$
\ln \left(\frac{I}{I_{0}}\right)=-L \sigma_{T} n=-\frac{L \sigma_{T}}{k T} p,
$$

where $I$ is the transmitted electron intensity, $I_{0}$ is the initial intensity, $n$ is the molecular gas density, $L$ is the interaction region length, $k$ is the Boltzmann constant, $T$ is the absolute temperature, and $p$ is the gas pressure. $T$ is derived from $T=\sqrt{T_{c} T_{m}}$, where $T_{c}$ and $T_{m}$ are the temperature of the scattering chamber measured with a thermocouple and the temperature of the Baratron gauge, respectively. Measurement conditions, data acquisition, and data analysis are controlled by a custom designed LabView (National Instruments) programme.

For each incident electron energy, attenuation measurements were repeated at least 5 times in order to ensure that statistical uncertainties remained below $4 \%$. Other random uncertainties are related to the temperature measurement (within $1 \%$, according to manufacturer's data) and the numerical fitting procedure (about 1\%). By combining these uncertainties, a total uncertainty limit of $5 \%$ has been determined for the present measurements. Systematic errors linked to the experimental technique are those connected to the so-called "missing angles (MA)."7 Due to the magnetic field confinement, the energy resolution determines the acceptance angle of the detector. As detailed in Ref. 7, and also in the work of Fuss et al. ${ }^{8}$ and Sanz et al. ${ }^{9}$ the magnitude of this systematic error can be evaluated from our theoretical data by integrating the calculated differential elastic and rotational excitation cross sections over the "missing" experimental angles. This effect is especially important for polar molecules, as is the case of thiophene $\left(\mu=0.52 \mathrm{D}^{10}\right)$. The significance of this error source in the present experimental results will be discussed in Sec. IV.

Prior to making the present thiophene total cross section measurements, the performance of the new apparatus and our measurement techniques were thoroughly benchmarked against the known TCS values of $\mathrm{N}_{2}{ }^{11,12}$ over the energy range of interest. Excellent agreement between our measured TCS data and the established values was found, giving us confidence in the validity of the TCS we have subsequently measured for thiophene.

\section{THEORETICAL METHODS}

As already mentioned, in order to cover the broad incident energy range considered in this study, we have used two different theoretical methods of proven reliability in their respective energy ranges of applicability. For the lower energies (1$15 \mathrm{eV})$, we have applied the R-matrix method ${ }^{13,14}$ within the fixed-nuclei approximation using the UKRmol suite. ${ }^{15}$ As usual, in order to include the contribution of the higher order partial waves and properly account for the dipole interaction, a Born approximation based method, implemented in the program POLYDCS, ${ }^{16}$ has been used to determine the differential and integral cross sections. The general features of these methods and the particular details on their application to thiophene have been published elsewhere. ${ }^{5}$ We note only that the approach used by POLYDCS introduces the rotational motion into the scattering and that therefore, the Borncorrected "elastic" cross section corresponds, in practice, to an electronically elastic one that includes rotational transitions from the rotational ground state $(\mathrm{J}=0)$ to rotational states with $0 \leq \mathrm{J} \leq 9$.

As discussed in Ref. 5, physical considerations lead us to recommend the Born-corrected close coupling (CC) results (that we will label R-matrix-CC-Born) as the most accurate of our R-matrix data. For intermediate and high energies (15$300 \mathrm{eV}$ ), we have used the latter version of our IAM-SCAR+I ${ }^{17}$ method together with an independent calculation based on the Born approximation (IAM-SCAR+I+R) to estimate the averaged dipole rotational excitation cross sections. ${ }^{18}$ Here we simply mention that including interference effects into the IAM-SCAR representation results in a clear increase in size of the differential elastic cross section for the smaller scattering angles, ${ }^{17}$ which consequently leads to an increase in the corresponding integral elastic cross sections (IECS). We have recently shown that for some benzene-like based molecules, such as pyridine, this increment can be of the order of $25 \%-30 \%$ for the higher energies. ${ }^{19}$ The IAM-SCAR procedure has provided reasonable agreement for a wide variety of molecular targets for energies above $\sim 20 \mathrm{eV},{ }^{20}$ but the role of the new interference terms still needs some further experimental validation. Inelastic scattering processes are not affected by the inclusion of the interference terms, and a single cross section for all inelastic processes is calculated from the imaginary part (absorption) of the interaction potential. However, as described in a recent article ${ }^{21}$ by alternately using as the threshold energy of the absorption potential either the minimum electronic-state excitation energy or the ionisation energy, we are able to extract the integral excitation and the integral ionisation cross sections from the calculated integral inelastic cross sections. We have recently shown that the total ionisation cross sections of some organic molecules, ${ }^{22,23}$ as derived from this procedure, are in fairly good agreement with the available experimental results.

\section{RESULTS AND DISCUSSION}

The total electron scattering cross sections, in SI units, measured with the experimental setup described above are shown in Table I together with our recent calculations using both the R-matrix-CC-Born and IAM-SCAR $+\mathrm{I}+\mathrm{R}$ procedures mentioned in Sec. III.

The absolute random uncertainties listed in Table I include the statistical fluctuation of direct measurements and the contribution of the sensitivity limits of all the electronic devices 
TABLE I. Present experimental electron scattering cross sections, TCS, their random uncertainty limits, the energy resolution ( $\triangle \mathrm{E})$, and the acceptance angle of the detector $(\Delta \theta)$ together with our R-matrix-CC-Born and IAM-SCAR+I+R calculations. ${ }^{5} \sigma(\Delta \theta)$ is the systematic error due to the acceptance angles as estimated with these calculations.

\begin{tabular}{|c|c|c|c|c|c|c|c|}
\hline \multirow[b]{2}{*}{ Energy (eV) } & \multicolumn{5}{|c|}{ Experiment } & \multicolumn{2}{|c|}{ Theory } \\
\hline & $\begin{array}{c}\text { TCS } \\
\left(\times 10^{-20} \mathrm{~m}^{2}\right)\end{array}$ & $\begin{array}{l}\text { Random uncertainty } \\
\qquad\left(\times 10^{-20} \mathrm{~m}^{2}\right)\end{array}$ & $\begin{array}{c}\Delta \mathrm{E} \\
(\mathrm{eV})\end{array}$ & $\begin{array}{c}\Delta \theta \\
(\operatorname{deg})\end{array}$ & $\begin{array}{c}\sigma(\Delta \theta) \\
\left(\times 10^{-20} \mathrm{~m}^{2}\right)\end{array}$ & $\begin{array}{c}\text { R-matrix } \\
\text { (CC-Born) }\end{array}$ & IAM-SCAR+I+R \\
\hline 1 & 27.9 & 0.9 & 0.21 & 27.3 & $19.6(10.2)^{\mathrm{a}}$ & 56.6 & 104 \\
\hline 1.2 & 33.1 & 0.3 & 0.22 & 25.3 & & 64.3 & \\
\hline 1.5 & 34.8 & 1.6 & 0.22 & 22.5 & $13.8(7.25)^{\mathrm{a}}$ & 62.4 & 85.4 \\
\hline 1.7 & 30.3 & 0.7 & 0.27 & 23.5 & & 63.4 & \\
\hline 2 & 34.8 & 1.2 & 0.21 & 18.9 & $10.2(6.33)^{\mathrm{a}}$ & 63.1 & 75.3 \\
\hline 2.2 & 37.8 & 0.9 & 0.21 & 18.0 & & 61.9 & \\
\hline 2.5 & 43.8 & 1.1 & 0.22 & 17.2 & & 60.8 & \\
\hline 2.7 & 46 & 0.7 & 0.24 & 17.3 & & 64.2 & \\
\hline 3 & 50.8 & 0.9 & 0.28 & 17.8 & $8.74(8.71)^{\mathrm{a}}$ & 66.4 & 66.9 \\
\hline 3.3 & 48 & 2.1 & 0.23 & 15.3 & & 56.1 & \\
\hline 3.6 & 47.9 & 0.9 & 0.22 & 14.3 & & 51.7 & \\
\hline 3.8 & 44.8 & 0.9 & 0.22 & 13.9 & & 49.9 & \\
\hline 4 & 43.4 & 1.4 & 0.23 & 13.9 & $6.63(4.86)$ & 48.6 & 63.6 \\
\hline 4.5 & 43.2 & 1.5 & 0.24 & 13.3 & & 46.7 & \\
\hline 5 & 47.1 & 1.1 & 0.24 & 12.7 & $6.03(4.20)^{\mathrm{a}}$ & 46.1 & 61.0 \\
\hline 5.5 & 47.4 & 1.6 & 0.26 & 12.5 & & 48.7 & \\
\hline 6 & 51.3 & 0.13 & 0.24 & 11.5 & & 49.4 & \\
\hline 6.5 & 47.3 & 0.9 & 0.23 & 10.8 & & 47.3 & \\
\hline 7 & 48.3 & 1.7 & 0.26 & 11.1 & $5.86(3.67)^{\mathrm{a}}$ & 46.2 & 59.6 \\
\hline 7.5 & 50.6 & 1 & 0.23 & 10.1 & & 47.1 & \\
\hline 8 & 51.7 & 1.3 & 0.2 & 9.10 & & 49.4 & \\
\hline 8.5 & 55.6 & 2.1 & 0.17 & 8.13 & & 51.0 & \\
\hline 9 & 53.6 & 1.9 & 0.27 & 9.97 & & 53.7 & \\
\hline 9.5 & 54 & 1.1 & 0.26 & 9.52 & & 54.5 & \\
\hline 10 & 58.1 & 1.6 & 0.25 & 9.10 & $4.96(3.43)^{\mathrm{a}}$ & 53.8 & 58.2 \\
\hline 11 & 58.3 & 1 & 0.25 & 8.67 & & 57.1 & \\
\hline 12 & 54.6 & 0.7 & 0.24 & 8.13 & & 57.7 & \\
\hline 13 & 61.4 & 0.8 & 0.23 & 7.64 & & 58.1 & \\
\hline 14 & 55.2 & 0.8 & 0.24 & 7.52 & & 58.4 & \\
\hline 15 & 54.8 & 0.3 & 0.24 & 7.27 & $3.90(3.43)^{\mathrm{a}}$ & 58.4 & 55.4 \\
\hline 16 & 52.3 & 0.6 & 0.21 & 6.58 & & & \\
\hline 17.5 & 49.5 & 1.1 & 0.27 & 7.13 & & & \\
\hline 20 & 49.3 & 1.1 & 0.27 & 6.67 & 4.14 & & 52.6 \\
\hline 25 & 46.9 & 1.4 & 0.19 & 5.00 & & & \\
\hline 30 & 47.5 & 1.8 & 0.19 & 4.56 & 2.66 & & 49.0 \\
\hline 40 & 43.3 & 1 & 0.22 & 4.25 & 2.07 & & 45.9 \\
\hline 50 & 38.9 & 0.4 & 0.22 & 3.80 & 2.02 & & 42.8 \\
\hline 70 & 35.4 & 0.2 & 0.17 & 2.82 & 1.49 & & 38.4 \\
\hline 100 & 33.1 & 0.3 & 0.19 & 2.50 & 1.38 & & 33.9 \\
\hline 150 & 29.9 & 1.2 & 0.21 & 2.14 & 0.47 & & 28.6 \\
\hline 200 & 26.9 & 0.3 & 0.26 & 2.07 & 0.66 & & 25.2 \\
\hline 250 & 22.8 & 0.8 & 0.22 & 1.70 & & & \\
\hline 300 & 19.8 & 0.8 & 0.22 & 1.55 & 0.75 & & 20.5 \\
\hline
\end{tabular}

${ }^{\mathrm{a} C a l c u l a t e d ~ w i t h ~ t h e ~ B o r n ~ c o r r e c t e d ~ R-m a t r i x ~ d i f f e r e n t i a l ~ c r o s s ~ s e c t i o n ~ v a l u e s . ~}$

used during the measurement procedure as well as those derived from the numerical data analysis. As the table shows, these uncertainties are within 5\% for all the scattering energies considered. However, we should note here that the main error source linked to transmission experiments such as those presented here is the aforementioned systematic error derived from the energy and angular resolution limits. The energy resolution of the present measurements is shown in Table I for each incident electron energy. These values are directly given by the electron intensity distribution of the incident beam as measured with the RPA in combination with the MCP detector (see Ref. 7 for details). As described in Ref. 7, under the present magnetic confinement conditions, the angular resolution $(\Delta \theta)$ is determined by the energy resolution $(\Delta E)$ according to the following expression:

$$
\Delta \theta=\arccos \sqrt{1-\frac{\Delta E}{E}} .
$$


The values of $\Delta \theta$ for each incident electron energy are also given in Table I. Before any comparison between the present experimental data and other available values can be made, an analysis of the energy and angular resolution limitations should be carried out in order to ensure a valid comparison.

In the present measurements, the angular and energy resolution limits make it impossible to detect elastically and rotationally scattered (the average rotational excitation energy is less than $1.1 \mathrm{meV})$ electrons within the $(0-\Delta \theta)$ and $(180-\Delta \theta)$ angular ranges. All these electrons are considered unscattered, thus lowering the measured TCSs. As shown in Ref. 7, the contribution of these "missing angles" $(\sigma(\Delta \theta))$ can be evaluated using the following expression:

$$
\begin{aligned}
\sigma(\Delta \theta)= & 2 \pi\left(\int_{0}^{\Delta \theta} \frac{d\left(\sigma_{e l}+\sigma_{r o t}\right)}{d \Omega} \sin \theta d \theta\right. \\
& \left.+\int_{180-\Delta \theta}^{180} \frac{d\left(\sigma_{e l}+\sigma_{r o t}\right)}{d \Omega} \sin \theta d \theta\right) .
\end{aligned}
$$

By using our IAM-SCAR+I+R and R-matrix-CC-Born differential cross sections (that include, as explained, rotational excitations), the contribution of the "missing angles" to the measured TCSs can be evaluated (MA correction). The results are shown in Table I. Note that these values always represent a contribution that would increase the observed TCS which may be taken into consideration when comparing with data from other sources.

For energies above $15 \mathrm{eV}$, where the IAM-SCAR+I+R method applies, there is good agreement (within 8.5\%) between the present measurements and the IAM-SCAR+I+R calculation. To illustrate the comparison, the present experimental and theoretical data are plotted in Fig. 1. As can be seen in this figure, if the rotational excitation is not included in the calculation (IAM-SCAR+I values), the agreement between experiment and theory is excellent. This is consistent with the fact that the energy and angular resolution used in most transmission experiments and, in particular, the present one

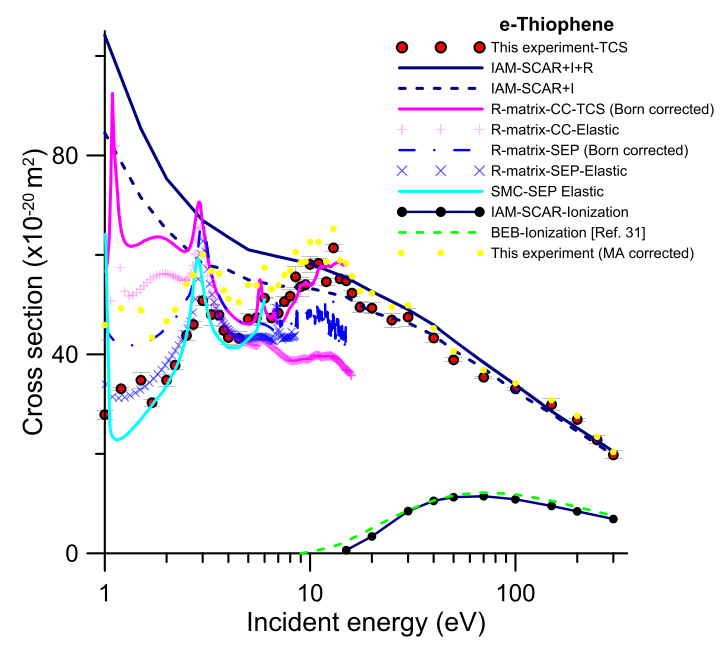

FIG. 1. Total electron scattering cross sections, including the present measurements, our IAM-SCAR+I+R, the R-matrix-SEP, and R-matrix-CC with Born correction results together with the R-matrix and SMCPP SEP and $\mathrm{R}$-matrix CC integral elastic cross sections without Born correction. Our IAMSCAR and the BEB (from Ref. 31) ionization cross sections are also shown. See also the legend on the figure. is not good enough to account for the rotational excitation processes, and comparisons between experimental and theoretical TCS values should be done excluding dipole rotation calculations. However, at these energies, the uncertainty due to the effect of the "missing angles" is less than $8.5 \%$. Combining this source of error with the random uncertainty limits, we consider the present experiment to provide reliable total electron scattering cross sections, within $10 \%$, in the energy range $10-300 \mathrm{eV}$. The excellent agreement between theory and experiment in this energy range also confirms the validity of introducing interference effects ${ }^{17}$ to the independent atom model (IAM) based calculations at intermediate and high energies. Furthermore, as explained in Ref. 17, including the interference term solves the contradiction between the optical theorem and the additivity rule (AR) assumed in such representations.

Below $10 \mathrm{eV}$, the IAM-SCAR+I+R method does not apply and comparison with this method does not make sense. At these lower energies, the comparison should be made with the reliable $a b$ initio R-matrix data. As shown in Table I, in the $2.1-15 \mathrm{eV}$ energy range we found a good agreement, within $10 \%$, between the experiment (including the "missing angle" correction) and the R-matrix-CC-Born results. Below this energy, from 1 to $2 \mathrm{eV}$, our calculation tends to give higher cross sections than the experimental values, reaching a maximum discrepancy of about $100 \%$ at $1 \mathrm{eV}$. However, we should note here that the R-matrix-CC-Born calculation includes rotational excitation which is not discernible by the experiment: as expected, these rotational excitations become more relevant for the lower energies. In fact, within the Born approximation, the rotational excitation cross sections increase exponentially for decreasing energies. Although we considered our CC the most accurate description of the scattering problem, ${ }^{5}$ Fig. 1 shows how our R-matrix-SEP-Born results agree better with the experimental values at these low energies but being still higher than those due to the Born corrected rotational excitations. The dominance of rotational excitations at low energies is illustrated in Fig. 2. Differential elastic and

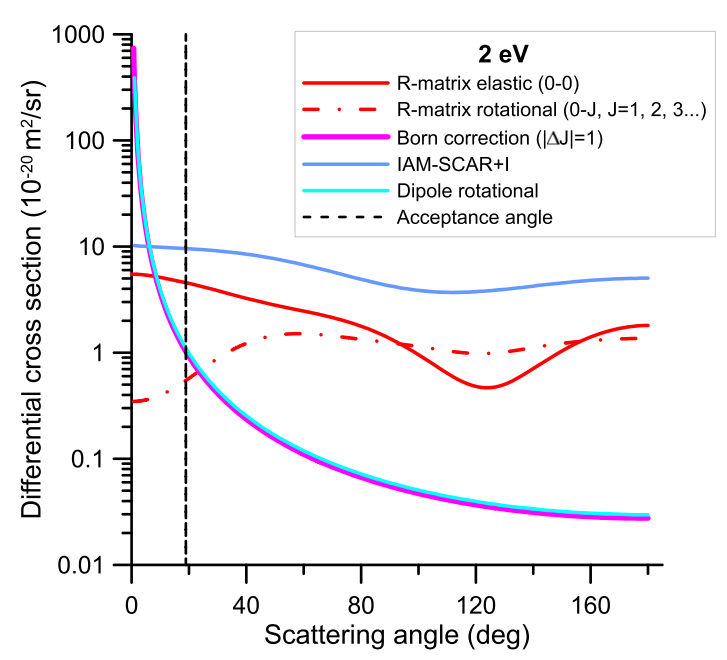

FIG. 2. Calculated differential elastic and rotational excitation cross sections for $2 \mathrm{eV}$ electron incident energy as a function of the scattering angle; the R-matrix data correspond to the SEP calculation. The acceptance angle of the detector for this incident energy is also shown. See also the legend on the figure. 
rotational excitation cross sections calculated with both our Rmatrix (at the SEP level) and IAM-SCAR $+\mathrm{I}+\mathrm{R}$ procedures are plotted for $2 \mathrm{eV}$ incident electron energy. The acceptance angle of the detector for this energy is also shown in Fig. 2 (dashed black line). The first feature we can distinguish in this figure is that the Born-based procedure used for our R-matrix (the POLYDCS ${ }^{16}$ formulation) and our dipole rotational excitation complementing the IAM-SCAR+I calculations are equivalent, leading to the same contribution to the DCS. It is also clear that the missing contribution to the cross section due to the acceptance angle limitation comes from the rotational excitation DCS and specifically the Born correction contribution, which are several orders of magnitude higher than the rotationally elastic DCS near $0^{\circ}$. The low energy failure of the IAM-SCAR+I calculation is also clearly explained by the rotationally elastic DCS. While this reproduces the angular dependence reasonably well, its absolute value is much higher (up to $100 \%$ ) than that given by the R-matrix calculation. However, the predominance of the Born correction at the lower energies justifies the good agreement found, even at very low energies, between both calculations for a highly polar molecule such as pyridine. $^{24}$

The above discussion shows the usefulness of comparing, for low energies, our experimental TCS with low energy scattering calculations not including the Born correction. Since below $8 \mathrm{eV}$ (not including rotations) elastic scattering processes are dominant, we have included in Fig. 1 our integral elastic (IECS) R-matrix data (both CC and SEP levels) ${ }^{5}$ and the elastic SMCPP ${ }^{6}$ calculation at the SEP level without inclusion of the Born correction. When this correction is excluded (and therefore the contribution of rotational excitation is mostly excluded), the CC-R-matrix IECS are closer, but still higher by about $60 \%$, to the experimental results. However, the SEP R-matrix IECS show a very good agreement with the experimental data. As described earlier, we have recently considered ${ }^{5}$ our CC approach as the most physical representation of the scattering problem and this is clearly the case above the first excitation threshold. ${ }^{5}$ However, for very low energies, due to the high molecular polarizability of thiophene $\left(60.8 \mathrm{a}_{\mathrm{o}}{ }^{3}\right),{ }^{25}$ the description of polarisation effects is crucial: it seems clear from Fig. 1 that the polarisation potential described by the SEP levels leads to the best agreement with the experimental results. This is also supported by the excellent agreement between the SMCPP calculation from da Costa et al. ${ }^{6}$ and the present experimental results.

Despite the difficulty involved in the comparison of low energy theoretical and experimental TCS data, this comparison can be very useful to validate some of the resonances predicted by different theories. These resonances appear as enhancement features in the experimental TCS and how well defined they are depends on the energy resolution (about $0.2 \mathrm{eV}$ in this experiment). In the case of thiophene, some of these features are clearly discernible below $15 \mathrm{eV}$ as shown in Fig. 1. As mentioned above, in this energy domain, our IAM-SCAR+I calculation does not apply and therefore its results are not going to be considered in this discussion. Both R-matrix ${ }^{5}$ and SMC-SEP ${ }^{6}$ calculations show a well-defined low energy resonance around $1 \mathrm{eV}$. da Costa et al. ${ }^{6}$ identified this peak as a $\pi^{*}$ shape resonance in the $B_{1}$ symmetry and their SEP calculation placed it at $1 \mathrm{eV}$. Our CC R-matrix calculation found a resonance at $1.1 \mathrm{eV}, 20 \%$ higher than the SEP approach. ${ }^{6}$ Our experimental TCS (see Fig. 1) shows a weak structure around $1.3 \pm 0.2 \mathrm{eV}$. Unfortunately, the energy resolution is not good enough to discriminate between calculations but this seems more compatible with a resonance at 1.1 than $1.0 \mathrm{eV}$ where our experimental results present a minimum. This is consistent with our statement that the $\mathrm{CC}$ calculation corresponds to the most complete representation of the scattering problem. The second peak, assigned by da Costa et al. ${ }^{6}$ to a superposition of a $\pi^{*}$ in the $A_{2}$ symmetry and a $\sigma^{*}$ shape resonance in the $B_{2}$ symmetry, is located at $2.82 \mathrm{eV}$ in their SEP calculation. ${ }^{6}$ The recent $\mathrm{R}$ matrix analysis of Loupas et al. ${ }^{26}$ located these resonances at $2.990 \mathrm{eV}$, the $\sigma^{*}\left(B_{2}\right)$, and $2.993 \mathrm{eV}$, the $\pi^{*}\left(A_{2}\right)$. The other R-matrix based calculation from Vinodkumar et al. ${ }^{27}$ located the $\sigma^{*}\left(B_{2}\right)$ resonance at $2.51 \mathrm{eV}$, although some inconsistencies of this assignment are discussed in Ref. 26. These values agree with the present experimental results which present a local maximum of the TCS at $3.0 \pm 0.2 \mathrm{eV}$. The position of these resonances as determined by our experiment is also consistent with the experimental vertical attachment energies of 1.15 and $2.63 \mathrm{eV}$, respectively, measured by Modelli and Burrow. ${ }^{28}$

Our experimental TCS values also show an increment around $3.3 \pm 0.2 \mathrm{eV}$ which may correspond to that observed by Muftakhov et al. ${ }^{29}$ at $3.5 \mathrm{eV}$, attributed by them to a Feshbachtype resonance whose parent state is the first excited triplet state of thiophene $\left({ }^{3} \mathrm{~B}_{2}\right)$. Core-excited resonances in thiophene have also been analysed by Loupas et al. ${ }^{26}$ by comparing the R-matrix calculation with experimental energy loss spectra. The two lowest lying resonances they found were identified as $1^{2} \mathrm{~A}_{2}$ at $5.695 \mathrm{eV}$ and $1^{2} \mathrm{~B}_{1}$ at $6.70 \mathrm{eV}$. The R-matrix calculation from Ref. 27 found the $1^{2} \mathrm{~A}_{2}$ resonance at $4.77 \mathrm{eV}$. Measurements of Ref. 29 observed the resonance at $5.3 \mathrm{eV}$. Our experimental TCS shows a shoulder around $5 \mathrm{eV}$ and a local maximum between 5.5 and 6.5 which is compatible with the position of these resonances when our energy resolution is taken into consideration. Experimental data from Ref. 29 and unpublished measurements cited in Ref. 26 (see Ref. 13 in Ref. 26) placed these resonances at 5.3, 5.38, 6.4, and $6.22 \mathrm{eV}$, respectively, in excellent agreement with the present measurements. In the energy range 6.9-9.5 eV, Loupas et al. ${ }^{26}$ identified 8 additional resonances. Only for two of them (a $2^{2} \mathrm{~B}_{1}$ at $7.96 \mathrm{eV}$ and a $2^{2} \mathrm{~A}_{2}$ at $9.22 \mathrm{eV}$ ) was any experimental evidence found, although discrepancies on the position of the latter are about $15 \%$. In this energy range, our experimental results only show a local maximum at $8.5 \mathrm{eV}$. This could be interpreted as a combination of the $2^{2} \mathrm{~B}_{1}$ resonance at 7.96 $\mathrm{eV}\left(1.2 \mathrm{eV}\right.$ width) with the $2^{2} \mathrm{~A}_{2}$ resonance at $9.22 \mathrm{eV}(0.95$ $\mathrm{eV}$ width) which is probably not well resolved by our experimental apparatus. The $8.5 \mathrm{eV}$ resonance was also observed by Muftakhov et al. ${ }^{29}$ which is identified in Ref. 27 as $2^{2} \mathrm{~A}_{2}$. The present experimental results finally show a broad maximum on the TCS values between 9.5 and $15 \mathrm{eV}$ presenting a weak peak at $11 \mathrm{eV}$ and the absolute maximum value at 13 $\mathrm{eV}$. This broad structure may be related to the excitation of the great number of accessible states, even those from the continuum (the ionisation threshold is $8.86 \mathrm{eV}$ ) and the overlap of 
numerous weak resonances not described by our methods. Above $15 \mathrm{eV}$, it presents a weak shoulder in the range $20-30 \mathrm{eV}$, probably a consequence of the combination between the decreasing elastic cross section with the still increasing excitation function of the mentioned excited states, and then monotonically decreases according to the energy dependence predicted by the IAM-SCAR+I theory. To summarize this comparison, the theoretical and experimental values of the positions of the above resonances are shown in Table II. Theoretical data from Kossoki and Bettega ${ }^{30}$ complementing those from Ref. 6 are also included in this table.

Finally, we also present in Fig. 1 the ionization cross sections we derived from the IAM-SCAR integral inelastic cross section (see Fig. 4 in Ref. 5) by using the alternate absorption threshold procedure mentioned in Sec. III (see Refs. 22 and 23 for details). The electron impact ionization cross section of

TABLE II. Position of the resonances observed in this study and those identified in previous experimental and theoretical publications.

\begin{tabular}{lcc}
\hline \hline Resonance & Experimental position $(\mathrm{eV})$ & Calculated position $(\mathrm{eV})$ \\
\hline$\pi_{1}^{*}\left(\mathrm{~B}_{1}\right)$ & $1.3 \pm 0.2^{\mathrm{a}}$ & $0.949^{26}(\mathrm{SEP})$ \\
& $1.15^{28}$ & $0.80^{26}(\mathrm{SEP})$ \\
& $1.114^{26}(\mathrm{CC})$ \\
& $1.00^{6,30}$ \\
\hline$\sigma^{*}\left(\mathrm{~B}_{2}\right)$ & $2.990^{26}(\mathrm{SEP})$ \\
& $2.51^{26}(\mathrm{SEP})$ \\
& $1.5^{26}(\mathrm{CC})$ \\
& $2.78^{6,30}$ \\
& $2.51^{27}$ \\
\hline$\pi_{2}^{*}\left(\mathrm{~A}_{2}\right)$ & $2.993^{26}(\mathrm{SEP} 35 \mathrm{VO})$ \\
& $2.87^{26}(\mathrm{SEP} 41 \mathrm{VO})$ \\
& $2.909^{26}(\mathrm{CC})$ \\
& $2.82^{6,30}$
\end{tabular}

\begin{tabular}{ccc}
\hline $1^{3} \mathrm{~B}_{2}$ & $3.3 \pm 0.2^{\mathrm{a}}$ & \\
& $3.5^{29}$ & \\
\hline $1^{2} \mathrm{~A}_{2}$ & $5.0 \pm 0.2^{\mathrm{a}}$ & $4.35^{27}$ \\
& $5.3^{29}$ & $5.695^{26}$ \\
& $5.38^{\mathrm{b}}$ & \\
\hline $1^{2} \mathrm{~B}_{1}$ & $6.0 \pm 0.2^{\mathrm{a}}$ & $6.70^{26}$ \\
& $6.4^{29}$ & \\
& $6.22^{\mathrm{b}}$ & \\
\hline
\end{tabular}

\begin{tabular}{ccc}
\hline $1^{2} \mathrm{~B}_{2}$ & $6.9^{26}$ \\
$2^{2} \mathrm{~B}_{2}$ & $7.72^{26}$ \\
$1^{2} \mathrm{~A}_{1}$ & $7.87^{26}$ \\
\hline $2^{2} \mathrm{~B}_{1}$ & $8.5 \pm 0.2^{\mathrm{a}}$ & $7.96^{26}$ \\
& $7.39^{\mathrm{b}}$ & \\
\hline
\end{tabular}

\begin{tabular}{lcc}
\hline $3^{2} \mathrm{~B}_{2}$ & & $8.98^{26}$ \\
$3^{2} \mathrm{~B}_{1}$ & & $9.01^{26}$ \\
\hline $2^{2} \mathrm{~A}_{2}$ & $8.5 \pm 0.2^{\mathrm{a}}$ & $9.22^{26}$ \\
& $8.5^{29}$ & $7.77^{27}$ \\
& $7.93^{\mathrm{b}}$ & \\
\hline
\end{tabular}

\begin{tabular}{lc}
\hline $2^{2} \mathrm{~A}_{1}$ & $9.48^{26}$ \\
\hline \hline
\end{tabular}

${ }^{\text {a Present experiment. }}$

${ }^{\mathrm{b}}$ Unpublished data (see Ref. 13 in Ref. 26). thiophene in the energy range 9-3000 eV was calculated by Mozejko et al. ${ }^{31}$ by using the binary-encounter-Bethe (BEB) model. ${ }^{32}$ The agreement between both calculations is fairly good except for energies around the ionisation limit where our IAM-SCAR method, due to the used independent atom representation, is not expected to be accurate. There are no experimental data available in the literature to compare these values so we believe that they provide a valuable complement for electron scattering databases.

\section{CONCLUSIONS}

Experimental electron scattering total cross sections for thiophene in the energy range $1-300 \mathrm{eV}$ have been measured for the first time with a magnetically confined electron transmission-beam apparatus. Total random uncertainty limits have been estimated to be within $5 \%$ by including the statistical reproducibility of the measurements and all the uncertainty sources connected to the measuring devices and data analysis procedures. The energy resolution has been measured directly from the transmission intensity distributions and found to be from 0.17 to $0.28 \mathrm{eV}$, depending on the incident electron energy. Systematic errors due to electrons elastically and rotationally scattered into the acceptance angle of the detector have been discussed in detail and evaluated with the help of calculated DCS values. Since the average rotational excitation energy of thiophene (about $0.001 \mathrm{eV}$ ) is much lower than the present energy resolution and the rotational excitation DCS are strongly peaked in the forward direction, the present experimental TCS data do not in practice account for the rotational excitation processes. Therefore looking at theoretical results without including Born corrections or dipole Born rotational excitation channels provides a more "like-with-like" comparison. When this is done, considering the mentioned random uncertainty limits and the angular limitations of the present experimental conditions, good agreement between the present measurements and our IAM-SCAR+I calculation has been found for energies above $15 \mathrm{eV}$. Below this value, excellent agreement has been found between the present experimental data and our R-matrix calculation at the $\mathrm{CC}$ level for energies between 3.6 and $15 \mathrm{eV}$. For lower energies, the dominant IECS has found to be extremely sensitive to the polarisation treatment included in the calculation procedure. Below these energies, even though the $\mathrm{CC}$ level can be considered the most complete representation of the scattering problem, we have found the experimental data to be in better agreement with calculations at the SEP level. With respect to the resonance positions, the present measurements confirmed the well-known low-lying $\pi^{*}$ and $\sigma^{*}$ shape resonances. Although most of the core-excited resonances identified in Ref. 26 are compatible with the present measurements, these do not have enough energy resolution to confirm their energy position and widths. One should also note that some of these coreexcited resonances may not enhance the elastic cross section significantly and may therefore not be visible in the TCS.

We consider that the present experimental values together with our previous calculation and those available in the literature constitute a reasonable electron scattering data set in the range $0-300 \mathrm{eV}$ ready to be used for modelling purposes. 
Future work to improve its accuracy should focus on the lower energy domain, mainly below $4 \mathrm{eV}$, improving the polarisation treatment in the R-matrix calculations to eliminate the discrepancy between CC and SEP results and on improving the energy resolution of the cross section measurements.

\section{ACKNOWLEDGMENTS}

This experimental study has been partially supported by the Spanish Ministerio de Ciencia, Innovación y Universidades (Project No. FIS 2016-80440) and the FP7-European UnionITN (Project No. 608163-ARGENT). A.I.L. also acknowledges the "Garantía Juvenil" grant programme from MINECO and support from the Doctorate Program in Science from the UNED University. J.D.G. acknowledges EPSRC funding.

${ }^{1}$ A. G. Sanz, M. C. Fuss, A. Muñoz, F. Blanco, P. Limão-Vieira, M. J. Brunger, S. J. Buckman, and G. García, Int. J. Radiat. Biol. 88, 71 (2012).

${ }^{2}$ H. Nikjoo, D. Emfietzoglou, T. Liamsuwan, R. Taleei, D. Liljequist, and S. Uehara, Rep. Prog. Phys. 79, 116601 (2016).

${ }^{3}$ R. D. White, D. Cooks, G. Boyle, M. Casey, N. Garland, D. Konovalov, B. Philippa, P. Stokes, J. de Urquijo, O. González-Magaña, R. P. McEachran, S. J. Buckman, M. J. Brunger, G. García, S. Dujko, and Z. Lj. Petrovic, Plasma Sources Sci. Technol. 27, 053001 (2018).

${ }^{4}$ Y. Zheng and L. Sanche, Appl. Phys. Rev. 5, 021302 (2018).

${ }^{5}$ A. Loupas, A. I. Lozano, J. D. Gorfinkiel, and G. García, J. Chem. Phys. 149, 034304 (2018).

${ }^{6}$ R. da Costa, M. do N. Varella, M. Lima, and M. Bettega, J. Chem. Phys. 138, 194306 (2013).

${ }^{7}$ A. I. Lozano, J. C. Oller, K. Krupa, P. Limão-Vieira, F. Blanco, A. Muñoz, R. Colmenares, and G. García, Rev. Sci. Instrum. 89, 063105 (2018).

${ }^{8}$ M. C. Fuss, A. G. Sanz, F. Blanco, J. C. Oller, P. Limão-Vieira, M. J. Brunger, and G. García, Phys. Rev. A 88, 042702 (2013).

${ }^{9}$ A. G. Sanz, M. C. Fuss, F. Blanco, J. D. Gorfinkiel, A. Almeida, F. Ferreira da Silva, P. Limão-Vieira, M. J. Brunger, and G. García, J. Chem. Phys. 139, 184310 (2013).

${ }^{10}$ A. McClellan, Tables of Experimental Dipole Moments (W. H. Freeman, 1963).
${ }^{11}$ C. Szmytkowski and K. Maciag, Phys. Scr. 54, 271 (1996).

${ }^{12}$ Y. Itikawa, J. Phys. Chem. Ref. Data 35, 31 (2006).

${ }^{13}$ J. Tennyson, Phys. Rep. 491, 29 (2010).

${ }^{14}$ P. G. Burke, R-Matrix Theory of Atomic Collisions: Application to Atomic, Molecular and Optical Processes (Springer, 2011).

${ }^{15}$ J. M. Carr, P. G. Galiatsatos, J. D. Gorfinkiel, A. G. Harvey, M. A. Lysaght, D. Madden, Z. Mašín, M. Plummer, J. Tennyson, and H. N. Varambhia, Eur. Phys. J. D 66, 20653 (2012).

${ }^{16}$ N. Sanna and F. Gianturco, Comput. Phys. Commun. 114, 142 (1998).

${ }^{17}$ F. Blanco, L. Ellis-Gibbings, and G. García, Chem. Phys. Lett. 645, 71 (2016).

${ }^{18}$ A. G. Sanz, M. C. Fuss, F. Blanco, F. Sebastianelli, F. A. Gianturco, and G. García, J. Chem. Phys. 137, 124103 (2012).

${ }^{19}$ A. Traoré Dubuis, F. Costa, F. Ferreira da Silva, P. Limão-Vieira, J. C. Oller, F. Blanco, and G. García, Chem. Phys. Lett. 699, 182 (2018).

${ }^{20}$ N. Hishiyama, M. Hoshino, F. Blanco, G. García, and H. Tanaka, J. Chem. Phys. 147, 224308 (2017).

${ }^{21}$ F. Blanco, F. Ferreira da Silva, P. Limão-Vieira, and G. García, Plasma Sources Sci. Technol. 26, 085004 (2017).

${ }^{22}$ W. A. D. Pires, K. L. Nixon, S. Ghosh, R. F. C. Neves, H. V. Duque, R. A. R. Amorim, D. B. Jones, F. Blanco, G. García, M. J. Brunger, and M. C. A. Lopes, Int. J. Mass Spectrom. 422, 32 (2017).

${ }^{23}$ S. Ghosh, K. L. Nixon, W. A. D. Pires, R. A. R. Amorim, R. F. C. Neves, H. V. Duque, M. G. M. da Silva, D. B. Jones, F. Blanco, G. García, M. J. Brunger, and M. C. A. Lopes, Int. J. Mass Spectrom. 430, 44 (2018).

${ }^{24}$ A. Sieradzka, F. Blanco, M. C. Fuss, Z. Mašín, J. D. Gorfinkiel, and G. García, J. Phys. Chem. A 118, 6657 (2014).

${ }^{25} \mathrm{Nist}$ standard reference database, edited by D. J. I. Russell, http://cccbdb.nist.gov; accessed 04 October 2017.

${ }^{26}$ A. Loupas, K. Regeta, M. Allan, and J. D. Gorfinkiel, J. Phys. Chem. A 122, 1146 (2018).

${ }^{27}$ M. Vinodkumar, H. Desai, and P. C. Vinokudmar, RSC Adv. 5, 24564 (2015).

${ }^{28}$ A. Modelli and P. D. Burrow, J. Phys. Chem. A 108, 5721 (2004).

${ }^{29}$ M. V. Muftakhov, N. L. Asfandiarov, and V. I. Khvostenko, J. Electron Spectrosc. Relat. Phenom. 69, 165 (1994).

${ }^{30}$ F. Kossoki and M. H. F. Bettega, J. Chem. Phys. 138, 234311 (2013).

${ }^{31}$ P. Możejko, E. Ptasińska-Denga, and Cz. Szmytkowski, Eur. Phys. J. D 66, 44 (2012).

${ }^{32}$ W. Hwang, Y.-K. Kim, and M. E. Rudd, J. Chem. Phys. 104, 2956 (1996). 